\title{
Relationship between age and prostate size
}

\author{
Shi-Jun Zhang ${ }^{1}$, Hai-Ning Qian ${ }^{2}$, Yan Zhao ${ }^{3}$, Kai Sun ${ }^{2}$, Hui-Qing Wang ${ }^{4}$, Guo-Qing Liang ${ }^{2}$, Feng-Hua Li $^{1}$ \\ and Zheng $\mathrm{Li}^{2}$
}

In a community-based study, the relationship between age and human prostate size was investigated in a population of men between the ages of 40 and 70 years to determine the normal prostate increase curve equation. One thousand male volunteers were randomly recruited from the Shanghai community, and the length, width, height, volume of the transition zone (TZ) and the whole prostates were measured by transrectal ultrasound (TRUS). Each volunteer was evaluated by the International Prostate Symptom Score (IPSS). Among those who completed the examination, the mean prostate parameters were all positively associated with increased age. There were statistically significant differences between each age group $(P<0.05)$. The mean transition zone volume (TZV) had a higher increase rate with age than the mean total prostate volume (TPV), indicating that the enlargement of the TZ contributed the most to the increase in TPV. While all prostate parameters were positively correlated with the IPSS, the strongest correlation was associated with the TZ length (TZL) and TZV. The growth curve equations for prostate width, height and length were also positively associated with increasing age.

Asian Journal of Andrology (2013) 15, 116-120; doi:10.1038/aja.2012.127; published online 10 December 2012

Keywords: benign prostate hyperplasia (BPH); growth equation; International Prostate Symptom Score (IPSS); prostate; transrectal ultrasound (TRUS)

\section{INTRODUCTION}

Benign prostatic hyperplasia (BPH) has been a considerable health problem to aging men for its associated symptoms and complications. Although not a life-threatening condition, BPH has a negative impact on a patient's quality of life, as evidenced in community and clinical studies. BPH is considered a progressive disease and its greatest risk factor for progression is age. ${ }^{1}$ Many studies have revealed a characteristic pattern of human prostatic growth with age. ${ }^{2-4}$ Bosch $e t$ al..$^{2}$ reported that the average percentage increases in total prostate volume (TPV) and transition zone volume (TZV) per year of follow-up were $2.2 \%$ and $3.5 \%$, respectively. Fukuta et al. ${ }^{5}$ conducted a cross-sectional community-based study to investigate the changes in TPV in Japanese men aged 40-80 years. The increase in prostate volume was measurable in each 10 -year age group and doubled from $5.5 \mathrm{ml}$ in $40-49$ years to $11.1 \mathrm{ml}$ in $70-80$ years.

While most of these studies were based on the TPV or TZV, the growth pattern or rate of growth of the total prostate and TZ was not investigated. Thus, the goal of this study was to investigate the increase rate pattern of the width, height and length of the total prostate and the TZ using transrectal ultrasound (TRUS), which made it possible to visualize the different zones. This study may help to identify men at increasing risk of $\mathrm{BPH}$ based on their prostate size and determine subsequent treatment management to prevent the progression of $\mathrm{BPH}$.

\section{MATERIALS AND METHODS}

\section{Participants}

This study was conducted from November 2009 to June 2010 in the Weifang Community in Shanghai, China, which has nearly 100000 permanent residents. All the permanent residents' personal information was available in the community office. The stratified cluster sampling method in a ratio of $10: 1$ was used to select 1000 men aged 40-70 years from the electoral rolls. A total of 864 men agreed to participate in the study, but 64 refused the TRUS. Out of the 800 men who participated, men who underwent radical prostatectomy and proctectomy, and who diagnosed with prostate cancer or were unable to complete the TRUS were excluded. At last, 762 men were involved in our study.

Prostate measurements and questionnaire

All the participants were invited to receive prostate measurement by TRUS with a 6-MHz uniplanar scanning probe (M-Turbo; SonoSite, Bothell, WA, USA), which was performed by an investigator who had 6 years of ultrasound experience. Under TRUS guidance, prostate width (maximal transverse diameter) was measured on an axial image, while prostate length (the longitudinal diameter as defined by the distance between proximal external sphincter and urinary bladder) and height (maximal antero-posterior diameter) were measured on a mid-sagittal image. ${ }^{6}$ The TPV was calculated with the prolate elliptical formula, $\pi / 6 \times$ width $\times$ height $\times$ length. ${ }^{6-9}$ The transition zone (TZ) was measured similarly to the above description. Length was defined as the greatest longitudinal distance on the sagittal scan, and width and height as the largest transverse and antero-posterior distance on axial scan. The TZV was also calculated with the prolate elliptical formula. ${ }^{10}$ 
Every participant was invited to perform a self-administered International Prostate Symptom Score (IPSS) questionnaire. Although a Chinese version of IPSS questionnaire was used, ${ }^{11}$ a urologist, who had 10 years working experience, explained the questionnaire to participants. ${ }^{12}$ The IPSS questionnaire was comprised of seven questions, which were stratified to determine the storage symptoms and voiding symptoms.

\section{Statistical analysis}

All the participants were divided into three groups by age: $40-49$ years, 50-59 years and 60-69 years. The analysis of variance was used to determine the significant differences in prostate size between the three age groups. The prostate increase model was established by the software Origin 8.0 (OriginLab, Northampton, MA, USA). A polynomial equation was used to fit the prostate increase model and the growthover-time equations. Correlations between the IPSS and prostate measurements were evaluated by Pearson's correlation coefficients. $P<0.05$ was considered statistically significant. Statistical analysis was performed with SPSS 13.0 (IBM; SPSS, Chicargo, IL, USA) for Windows.

\section{RESULTS}

A total of 762 men participated in the study. They were divided into groups by age: 106 in $40-49$ years group, 335 in 50-59 years group and 321 in $60-70$ years old group. The mean TPVs were increased significantly $(P=0.0023)$. The mean TZVs for the three groups were also significantly different among the groups $(P<0.05)$. Table 1 shows the mean width, height, length of total prostate and TZ parameters in each age group. Among the three age groups, all parameters increased significantly with age $(P<0.05)$, indicating a positive correlation between prostate size and age.

The percentage increase in prostate size ranged from $2.0 \%$ to $13.6 \%$ for most parameters. To determine the rate at which prostate parameters increased with age, the following equations were calculated: for width increase rate, $Y=1.6 \times 10^{-5} x^{3}-0.0021 x^{2}+0.0746 x+0.6772$; for length increase rate, $Y=1.6 \times 10^{-5} x^{3}-0.0018 x^{2}+0.073 x-0.6909$; and for height increase rate, $Y=-2.4 \times 10^{-5} x^{3}+0.0033 x^{2}-0.1312 x+$ 1.269 , where $x=$ age (40-70 years) (Figure 1). With these growth rate equations, we determined that prostate width and height had relatively stable growth rates compared to prostate height in men aged 40-70 years. However, in 60-70 years group, the growth rate as measured by the length increase, exceeds the rate of the width increase. Thus, the mean TZV per age group increased faster than the mean TPV, and mean length per age group increased the fastest after the age of 60 years.

All of the participants in our study completed the IPSS questionnaire. Table 2 shows the relationship between the IPSS and the width,

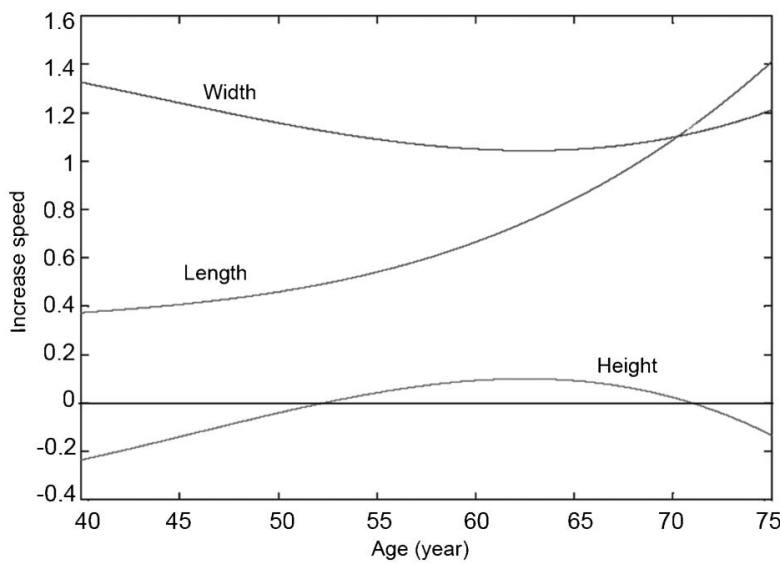

Figure 1 Rate of increase in prostate measurements over time.

height and length of the total prostate and TZ. Upon statistical analysis, each measurement was significantly positively correlated with the total IPSS storage score and voiding score $(P<0.001)$. The TZ length (TZL) and the TZV had the strongest significant correlation with the IPSS ( $r=0.319$ and 0.328 , respectively; Figure 2 ). The correlation between storage symptom scores and the TZL and the TZV were slightly stronger than that between voiding symptom scores and TZL ( $r=0.284,0.303 v s .0 .273,0.288$, respectively). In addition, TZV had a relatively strong relationship with the IPSS question regarding a weak stream.

Using a polynomial equation, the parameters data were used to determine the prostate growth rate curve (Figure 3 ). The followings are the growth rate increase curve equations for total prostate width, height and length, respectively: $Y=4 \times 10^{-6} x^{4}-0.0007 x^{3}+0.0373 x^{2}-$ $0.6772 x+44, Y=-6 \times 10^{6} x^{4}+0.0011 x^{3}-0.0656 x^{2}+1.269 x+32, Y=4 \times 10^{-6}$ $x-0.0006 x^{4}+0.0365 x^{3}-0.69 .9 x^{2}+36$, where $x=$ age ( $40-70$ years). Thus, these equations could calculate the mean prostate measurements on the basis of age.

\section{DISCUSSION}

Benign prostatic enlargement is the most common reason for lower urinary tract symptoms and bladder outlet obstruction, and is also an important determinant of BPH. Prostate volume is a crucial preoperative evaluation for prostate surgery. TRUS is a non-invasive and inexpensive method for measuring different regions of the prostate. Sech et al. ${ }^{13}$ reported that the reliability of TRUS for measuring total prostate and TZV was high for examiners with different levels of experience, although intra- and interobserver variability of TRUS in

Table 1 Various prostate measurements (mean \pm s.d.)

\begin{tabular}{|c|c|c|c|c|c|c|}
\hline Age & $\begin{array}{c}\text { Group } 1 \\
\text { (40-49 years) }\end{array}$ & $\begin{array}{c}\text { Group } 2 \\
\text { (50-59 years) }\end{array}$ & $\begin{array}{c}\text { Increase from } \\
\text { Group } 1 \text { to Group } 2 \\
\text { (\%) }\end{array}$ & $\begin{array}{c}\text { Group } 3 \\
\text { (60-70 years) }\end{array}$ & $\begin{array}{c}\text { Increase from } \\
\text { Group } 2 \text { to Group } 3 \\
\text { (\%) }\end{array}$ & $P$ \\
\hline Width (mm) & $44.45 \pm 4.67$ & $45.53 \pm 4.70$ & 2.4 & $46.69 \pm 5.10$ & 2.5 & 0.0134 \\
\hline Height (mm) & $31.66 \pm 4.38$ & $32.34 \pm 4.60$ & 2.1 & $33.50 \pm 5.91$ & 3.6 & 0.0471 \\
\hline Length (mm) & $37.55 \pm 4.27$ & $38.89 \pm 4.64$ & 3.5 & $41.13 \pm 6.18$ & 5.7 & 0.0002 \\
\hline TPV (mm) & $28.17 \pm 8.75$ & $30.83 \pm 9.64$ & 9.4 & $35.03 \pm 17.41$ & 13.6 & 0.0023 \\
\hline TZW (mm) & $30.25 \pm 4.84$ & $31.92 \pm 5.15$ & 5.5 & $34.21 \pm 7.08$ & 7.2 & 0.0001 \\
\hline TZH (mm) & $21.64 \pm 3.73$ & $23.24 \pm 4.58$ & 7.4 & $25.02 \pm 6.07$ & 7.7 & 0.0001 \\
\hline TZL (mm) & $25.24 \pm 4.03$ & $27.20 \pm 4.46$ & 7.8 & $29.43 \pm 6.24$ & 8.2 & $<0.0001$ \\
\hline TZV (mm) & $8.95 \pm 3.88$ & $11.15 \pm 5.95$ & 24.6 & $14.92 \pm 13.49$ & 33.8 & 0.0001 \\
\hline
\end{tabular}

Abbreviations: TPV, total prostate volume; TZH, transition zone height; TZL, transition zone length; TZV, transition zone volume; TZW, transition zone width. 
Table 2 Pearson's correlations of prostate measurements with IPSS

\begin{tabular}{|c|c|c|c|c|c|c|c|c|}
\hline & Width & Length & Height & $T P V$ & $T Z W$ & $T Z L$ & $T Z H$ & $T Z V$ \\
\hline Q1 Incomplete emptying & 0.104 & 0.114 & 0.133 & 0.160 & 0.149 & 0.145 & 0.163 & 0.191 \\
\hline Q2 Frequency & 0.075 & 0.139 & 0.171 & 0.171 & 0.191 & 0.190 & 0.208 & 0.232 \\
\hline Q3 Intermittency & 0.120 & 0.121 & 0.121 & 0.153 & 0.158 & 0.131 & 0.158 & 0.175 \\
\hline Q5 Weak stream & 0.196 & 0.187 & 0.221 & 0.230 & 0.262 & 0.262 & 0.270 & 0.271 \\
\hline Q6 Straining & 0.161 & 0.176 & 0.194 & 0.207 & 0.262 & 0.234 & 0.259 & 0.253 \\
\hline Q7 Nocturia & 0.078 & 0.175 & 0.167 & 0.185 & 0.211 & 0.236 & 0.237 & 0.258 \\
\hline Total IPSS & 0.168 & 0.212 & 0.195 & 0.241 & 0.291 & 0.319 & 0.279 & 0.328 \\
\hline
\end{tabular}

Abbreviations: IPSS, International Prostate Symptom Score; TPV, total prostate volume; TZH, transition zone height; TZL, transition zone length; TZV, transition zone volume; TZW, transition zone width.

prostate volume measurements has also been reported. ${ }^{14}$ Although the 3D TRUS images had lower variability and higher reliability than that of $2 \mathrm{D}$ images, the 2D TRUS has been the preferred method for estimating prostate volume as 3D TRUS is labor-intensive, time-consuming and tedious to perform. ${ }^{10,14}$ Therefore, the TRUS is a reliable method for measuring the prostates.

The size of the prostate typically increases throughout a man's lifetime. ${ }^{15,16}$ Different growth characteristics in each prostate zone may contribute to differences in the overall growth rate with age. The morphology and pathology of each prostate zone has been well described. ${ }^{17}$ However, little is known about the growth characteristics of each prostate region. Understanding prostate growth characteristics is helpful for the diagnosis and management of early stage $\mathrm{BPH}$, which perhaps could delay its progression. One of the goals of this study was to use TRUS to investigate the changes in total prostate and TZVs with age.

The greatest increases in size were observed in the TZ and TPV parameters and were significantly different between the age groups. Increases in prostate width, height and length were also associated with increased age but to a lesser extent than found in the TZ. In a similar report by Bosch et al., ${ }^{2}$ measured prostate volumes with TRUS at baseline after 2.1 and 4.2 years in a community-based study and

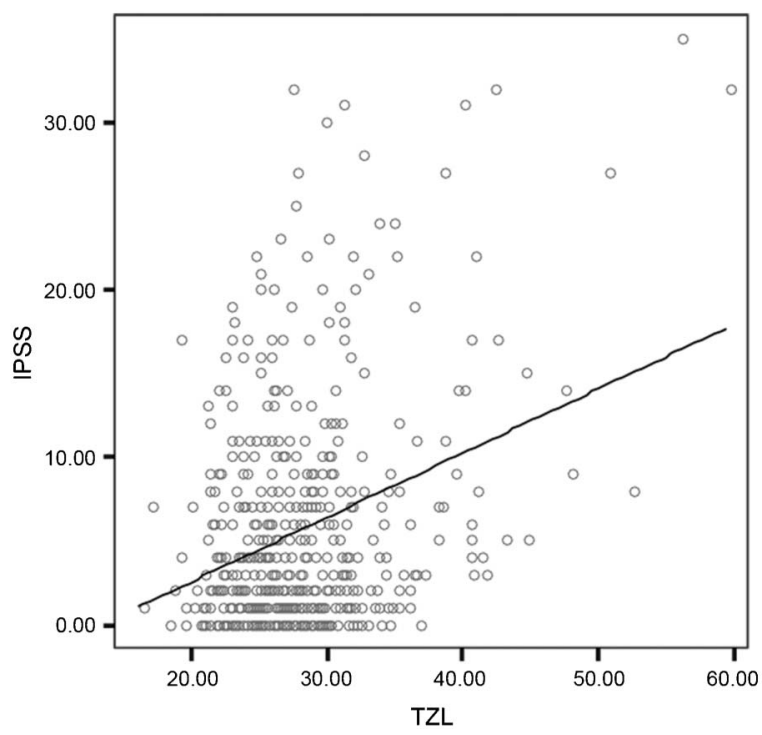

Figure 2 Correlation coefficient between the transition zone length (TZL) and the International Prostate Symptom Score (IPSS) $(r=0.319)$.
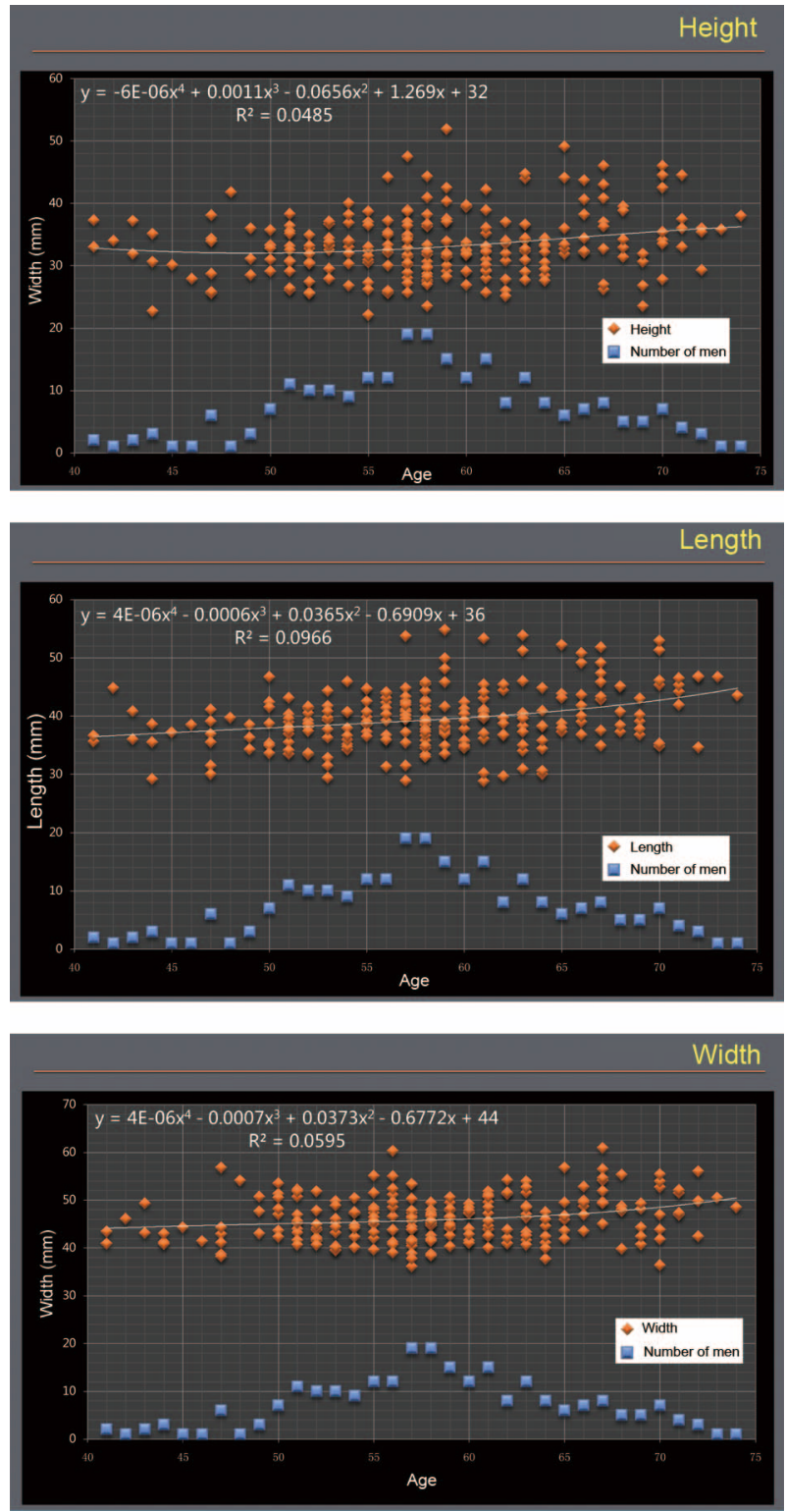

Figure 3 Increase curves of prostate width, height and length. 
found that the average percentage increases in TPV and TZV per year of follow-up were $2.2 \%$ and $3.5 \%$, respectively. Their results were similar to the results from this study, where the percentage increase rates were found to be $9.4 \%$ and $13.6 \%$ for the age groups of $40-49$ and 50-59 years, respectively. Similarly, Rhodes et al. ${ }^{3}$ found that the baseline prostate volume was a good predictor for growth rate, and determined that while there was wide variability in prostate growth rate on an individual level, prostate volumes appeared to increase steadily at about $1.6 \%$ per year in a random population.

By comparing the percentage increase in prostate parameters in the three age groups, the increase in the TZV was higher than that of the total prostate. This demonstrates that the TZ growth was the main contributor to the total prostate growth. Additionally, the peripheral zone atrophied in many BPH patients. These changes in the TZ could be attributed to several factors including changes in androgen levels and changes in nodular size. ${ }^{17,18}$

From these parameters, prostate width and height had a relatively stable rate of increase and changed little among men aged 40-49 and $50-59$ years. However, after 60 years old, the rate of increase in prostate length increased, creating an increase in prostate diameter. This shows that before the age of 60 years, the prostate grows slowly, while after the age of 60 years, the length becomes the main growth line. Intravesical prostatic protrusion has been implicated in the increase in prostate length. Han et al. ${ }^{19}$ found that the degree of intravesical prostatic protrusion was positively correlated with age $(r=0.210$; $P<0.01)$ and an increase in intravesical prostatic protrusion contributed to the prostate length increase, causing bladder outlet obstruction (BOO) in BPH patients. ${ }^{19-21}$ Another report by Guan et al. ${ }^{22}$ explored the correlation between the parameters of prostate volume and urodynamic results in diagnosing BOO. By logistic regression analysis, only the length, prostate volume and maximum flow rate were found to be positively correlated with Schafer grading value. Therefore, the length of the prostate could be used to evaluate the BOO level. These findings are in accordance with our results.

Studies examining the correlation between prostate volume and IPSS have been mixed. A few studies have shown a positive and statistically significant correlation. ${ }^{23-25}$ Wang et al. ${ }^{26}$ reported a correlation coefficient of 0.27 between the prostate volume and the IPSS in a Chinese survey, which is similar to the correlation found in this study. Conversely, no correlation was found between TPV, TZV and the prostate symptoms score evaluated by the IPSS in a study developed by Kaplan et al. ${ }^{27}$ The discrepancy is that these studies are likely the result of different study populations and methods. The TZL had a better correlation with IPSS than TPV. Similar results were also found by Lee et al. ${ }^{28}$ in a study that showed that TZL was strongly correlated with the IPSS ( $r=0.266$ ), even though the length of the total prostate was not. It seems that the most important dimensional parameter in prostates is the longitudinal diameter of the TZ, which travels in the same direction as the urethra. It is possible that the faster increase in length is a high risk factor for the $\mathrm{BOO}$ after the age of 60 years.

The storage score had a slightly stronger relationship with TZL, $\mathrm{TZH}$ and TZV than the voiding score. It has been suggested that the increase in TZL, TZH and TZV would be more likely to cause negative storage symptoms than voiding symptoms, but the correlation coefficient differed little. Some research has shown that there was no significant difference between the two IPSS subscores. ${ }^{26}$ However, Ezz el Din et al. ${ }^{29}$ found that the filling IPSS score had significant correlation with prostate volume and voiding score, while the total IPSS had not. In this study, only the IPSS question regarding a weak stream had relatively higher correlation with the size of the TZ, and this question was included in voiding symptoms. The increase in the size of the TZ is likely a significant cause of weak urinary stream.

With these parameters of the prostate, the volume growth curve equation was fitted to establish the prostate growth model. The human prostate has an age-stratified growth pattern; it grows at different rates in different age stages. Establishing the prostate increase equation could be used as the basis of treatment and monitoring the effect of $\mathrm{BPH}$. A study by Xia et al. ${ }^{16}$ pointed out that the age-stratified growth of the human prostate could be categorized into four life stages with the second rapid growing phase from 50 to 90 years and the prostate growth rate of $0.5-1.2$ g per year. In this study, the sample age was similar to the second rapid growing phase age and the equation was fitted for an increase model for the ages 40-70 years. One study noted that the overall prostatic growth increases with age and peaks with an increase of $4.15 \pm 4.98 \mathrm{ml}$ per year between the ages of 56 and 65 years and declines rapidly thereafter. ${ }^{30}$ Based on our results from the equation of increase curve, it is possible to calculate the mean prostate size of volume, width, height and length in a man of any age between the ages of 40 and 70 years.

A report from a community-based study in the Netherlands demonstrated that the mean prostate volume in every age group was larger than that of our study. ${ }^{2}$ Another study showed that the prevalence of digital rectal examination-detected enlarged prostate in West African men was higher than previously reported for American men, but the prevalence of lower urinary tract symptoms was lower than previously reported for African Americans. ${ }^{31}$ These differences in prostate volume are likely due to cultural differences; as such, the equations may only be useful in a Chinese population or one with similar cultural demographics.

A limitation of this study was that prostate parameters were taken only at a single time point. The best way to monitor the prostate growth pattern would be to perform a longitudinal study, where all of the participants could be measured and followed for several years in order to obtain an accurate growth rate. This study was a crosssectional observation of men from Shanghai, and future longitudinal studies are warranted to confirm the present study results.

\section{CONCLUSION}

Larger prostate volumes and TZV were positively associated with increased age, and the mean TZV per age group increased at a rate faster than the TPV. The mean length of prostate increased faster than the height and width, especially after the age of 60 years. The TZV and TZL had a higher correlation with IPSS, indicating a link between prostate size and symptoms of BPH. Using the width, height and length growth rate equations, the mean size of the prostate could be calculated in any man aged 40-70 years. These equations and models can facilitate further studies about prostate growth and may enable early diagnosis of $\mathrm{BPH}$.

\section{AUTHOR CONTRIBUTIONS}

SJZ, FHL and ZL designed this study, and SJZ wrote the draft of the manuscript. YZ and SJZ performed the statistical analysis. SJZ, GQL, HQW and KS participated in data collection, and assisted in manuscript elaboration. All authors read and approved the final manuscript.

\section{COMPETING FINANCIAL INTERESTS}

The authors declare that they have no competing financial interests.

\section{ACKNOWLEDGMENTS}

The project was supported by the Major Scientific Research Proposal of the Science and Technology Commission of Shanghai Municipality 
(No. 09DJ1400400). This work was also supported by Foundation of Pudong Medical Technology (No. PW2011D-1) and Shanghai Excellent Young Teachers Program (No. jdy11064) to Shi-Jun Zhang.

1 Emberton M, Andriole GL, de la Rosette J, Djavan B, Hoefner K et al. Benign prostatic hyperplasia: a progressive disease of aging men. Urology 2003; 61: 267-73.

2 Bosch JL, Tilling $\mathrm{K}$, Bohnen AM, Bangma $\mathrm{CH}$, Donovan JL. Establishing normal reference ranges for prostate volume change with age in the population-based Krimpen-study: prediction of future prostate volume in individual men. Prostate 2007; 67: 1816-24.

3 Rhodes T, Girman CJ, Jacobsen SJ, Roberts RO, Guess HA et al. Longitudinal prostate growth rates during 5 years in randomly selected community men 40 to 79 years old. J Urol 1999; 161: 1174-9.

4 Loeb S, Kettermann A, Carter HB, Ferrucci L, Metter EJ et al. Prostate volume changes over time: results from the Baltimore Longitudinal Study of Aging. J Urol 2009; 182: 1458-62.

5 Fukuta F, Masumori N, Mori M, Tsukamoto T. Internal prostatic architecture on transrectal ultrasonography predicts future prostatic growth natural history of prostatic hyperplasia in a 15-year longitudinal community-based study. Prostate $2011 ; 71$ : 597-603.

6 Jeong CW, Park HK, Hong SK, Byun SS, Lee HJ et al. Comparison of prostate volume measured by transrectal ultrasonography and MRI with the actual prostate volume measured after radical prostatectomy. Urol Int 2008; 81: 179-85.

7 Lee JS, Chung BH. Transrectal ultrasound versus magnetic resonance imaging in the estimation of prostate volume as compared with radical prostatectomy specimens. Urol Int 2007; 78: 323-7.

8 Aarnink RG, de la Rosette JJ, Debruyne FM, Wijkstra H. Formula-derived prostate volume determination. Eur Urol 1996; 29: 399-402.

9 Bangma CH, Niemer AQ, Grobbee DE, Schröder FH. Transrectal ultrasonic volumetry of the prostate: in vivo comparison of different methods. Prostate 1996; 28: 107-10.

10 Zlotta AR, Djavan B, Damoun M, Roumeguere T, Petein M et al. The importance of measuring the prostatic transition zone an anatomical and radiological study. BJU Int 1999; 84: 661-6.

11 Shao Q, Guo YW, Guo HB, Zhang YH. [An evaluation of the chinese version of international prostate symptom score]. Chin J Urol 2001; 22: 51-3.

12 Oztürk Mî, Koca O, Keleş MO, Güneș M, Kaya C et al. International prostate symptom score: really appreciated by all patients or not? Urol J 2011; 8: 227-30.

13 Sech S, Montoya J, Girman CJ, Rhodes T, Roehrborn CG. Interexaminer reliability of transrectal ultrasound for estimating prostate volume. J Urol 2001; 166: 125-9.

14 Tong S, Cardinal HN, McLoughlin RF, Downey DB, Fenster A. Intra- and inter-observer variability and reliability of prostate volume measurement via two-dimensional and three-dimensional ultrasound imaging. Ultrasound Med Biol 1998; 24: 673-81.
15 Berry SJ, Coffey DS, Walsh PC, Ewing LL. The development of human benign prostatic hyperplasia with age. J Urol 1984; 132: 474-9.

16 Xia SJ, Xu XX, Teng JB, Xu CX, Tang XD. Characteristic pattern of human prostatic growth with age. Asian J Androl 2002; 4: 269-71.

17 McNeal JE. The zonal anatomy of the prostate. Prostate $1981 ; 2$ : 35-49.

18 Selman SH. The McNeal prostate: a review. Urology 2011; 78: 1224-8.

19 Han WK, Shan GZ, Jin J. Correlation of intravesical prostatic protrusion with clinical evaluation parameters in BPH patients. Zhonghua Nan Ke Xue 2010; 16: 254-7.

20 Park SC, Lee JW, Rim JS. The relationship between intravesical prostatic protrusion and pressure flow study findings in patients with benign prostate obstruction/lower urinary tract symptoms. Actas Urol Esp 2012; 36: 165-70.

$21 \mathrm{Kim} \mathrm{KH}$, Kim YS. Correlation of male overactive bladder with intravesical prostatic protrusion. Korean J Urol 2010; 51: 843-6.

22 Guan YJ, Pang ZL, Wei J, Cai ZT, Sun CQ. [Clinical significance of parameters of prostate volume measured by transabdominal ultrasonography in evaluating bladder outlet obstruction]. Zhonghua Yi Xue Za Zhi 2008; 88: 977-9.

23 Franciosi M, Koff WJ, Rhoden EL. Correlation between the total volume, transitional zone volume of the prostate, transitional prostate zone index and lower urinary tract symptoms (LUTS). Int Urol Nephrol 2007; 39: 871-7.

24 Overland GB, Vatten L, Rhodes T, DeMuro C, Jacobsen G et al. Lower urinary tract symptoms, prostate volume and uroflow in Norwegian community men. Eur Urol 2001; 39: 36-41.

25 Bosch JL, Hop WC, Kirkels WJ, Schröder FH. The International Prostate Symptom Score in a community-based sample of men between 55 and 74 years of age: prevalence and correlation of symptoms with age, prostate volume, flow rate and residual urine volume. Br J Urol 1995; 75: 622-30.

26 Wang JY, Liu M, Zhang YG, Zeng P, Ding $Q$ et al. Relationship between lower urinary tract symptoms and objective measures of benign prostatic hyperplasia: a Chinese survey. Chin Med J (Engl) 2008; 121: 2042-5.

27 Kaplan SA, Reis RB, Staimen VB, Te AE. Is the ratio of transition zone to total prostate volume higher in African-American men than in their Caucasian or Hispanic counterparts? Br J Urol 1998; 82: 804-7.

28 Lee T, Seong DH, Yoon SM, Ryu JK. Prostate shape and symptom score in benign prostatic hyperplasia. Yonsei Med J 2001; 42: 532-8.

29 Ezz el Din K, Kiemeney LA, de Wildt MJ, Debruyne FM, de la Rosette JJ. Correlation between uroflowmetry, prostate volume, postvoid residue, and lower urinary tract symptoms as measured by the International Prostate Symptom Score. Urology 1996; 48: 393-7.

30 Williams AM, Simon I, Landis PK, Moser C, Christens-Barry W et al. Prostatic growth rate determined from MRI date: age-related longitudinal changes. J Androl 1999; 20: 474-80.

31 Chokkalingam AP, Yeboah ED, Demarzo A, Netto G, Yu K et al. Prevalence of BPH and lower urinary tract symptoms in West Africans. Prostate Cancer Prostatic Dis 2012; 15: $170-6$. 